\title{
Fungal polysaccharides: biological activity beyond the usual structural properties
}

\author{
Marcio L. Rodrigues ${ }^{1}{ }^{*}$, Leonardo Nimrichter ${ }^{1}$, Radames J. B. Cordero ${ }^{2}$ and Arturo Casadevall ${ }^{2,3}$ \\ 1 Laboratório de Estudos Integrados em Bioquímica Microbiana, Instituto de Microbiologia Professor Paulo de Góes, Federal University of Rio de Janeiro, Rio de \\ Janeiro, RJ, Brazil \\ ${ }^{2}$ Department of Microbiology and Immunology, Albert Einstein College of Medicine, Bronx, NY, USA \\ ${ }^{3}$ Department of Medicine, Albert Einstein College of Medicine, Bronx, NY, USA
}

Edited by:

Joshua D. Nosanchuk, Albert Einstein

College of Medicine, USA

Reviewed by:

Joshua D. Nosanchuk, Albert Einstein

College of Medicine, USA

Oscar Zaragoza, Instituto de Salud

Carlos III, Spain

*Correspondence:

Marcio L. Rodrigues, Avenida Carlos

Chagas Filho 373, CCS, Bloco I.

Instituto de Microbiologia, Federal

University of Rio de Janeiro, Rio de

Janeiro, RJ 21941-902, Brazil.

e-mail:marcio@micro.ufrj.br
Studies on structure and function of polysaccharides in biological systems classically involve sequence and compositional analyses, anomeric configuration, type of glycosidic linkage, and presence of substituents. Recent studies, however, indicates that other structural parameters, so far little explored, can directly influence the biological activity of microbial polysaccharides. Among these parameters, we highlight the molecular dimensions of Cryptococcus neoformans polysaccharides, which appear to be inversely correlated with their immunobiological activity. These recent observations raise new concepts about the structure and function of polysaccharides, which stimulates the design of new experimental approaches and suggests previously unknown applications.

Keywords: polysaccharides, fungal pathogens, immunity, structure and function of polysaccharides, Cryptococcus neoformans
Polysaccharides are cellular structural components found in the three domains of life. In microorganisms, several studies have shown that polysaccharides play crucial roles in the architecture of the cell envelope (Roberts, 1996; Nimrichter et al., 2005). In both prokaryotic and eukaryotic microbial pathogens, polysaccharides are major cell wall components (Roberts, 1996; Nimrichter et al., 2005). This surface distribution is in agreement with the fact that polysaccharide molecules directly influence host-pathogen interactions (Moxon and Kroll, 1990; Smith, 1990; Levitz, 2004; Zaragoza et al., 2009).

Polysaccharides consist of polymeric structures composed of at least ten monosaccharides sequentially connected by glycosidic bonds (Bertozzi and Rabuka, 2009; Mulloy et al., 2009; Stanley and Cummings, 2009). These structures may be linear or branched, a characteristic that is observed when a monosaccharide constituent of a polysaccharide is involved in more than two glycosidic bonds. Polysaccharides can be classified as homopolymers, a term used to indicate a polymer composed of identical monosaccharides, or heteropolymers, a term used for classification of polysaccharides composed of two or more types of monosaccharides. Classic approaches for the determination of polysaccharide structure include chromatographic methods in association with spectrometric and spectroscopic techniques. These approaches allow determination of sequence and composition, anomeric configuration, type of glycosidic linkage, and presence of substituents (Mulloy et al., 2009).

Capsular polysaccharides were amongst the earliest microbial virulence determinants described in the literature, as demonstrated in the classic Griffith's experiment [review in (Smith, 1990)]. This pioneering study established a direct relationship between the presence of polysaccharide capsules in bacterial pathogens and protection against host defenses. Subsequently, over several decades, the association between microbial virulence and capsular polysaccharides has been consolidated (Moxon and Kroll, 1990; Monari et al., 2006; Vecchiarelli, 2007; Zaragoza et al., 2009), although it is clear that in some cases, these structures work in favor of the host (Mazmanian and Kasper, 2006; Pletz et al., 2008; Kumar et al., 2009). Consequently, microbial polysaccharides may work in favor of the pathogen or induce immune responses that promote infection control, depending on their chemistry and/or structural aspects.

Classically, polysaccharide antigens have been considered poor inducers of cellular immunity. In fact, polysaccharide molecules are considered T-cell independent antigens that are more efficient activators of antibody production than of cell-mediated immune responses (Weintraub, 2003). In the last decade, however, a number of studies have demonstrated the role of polysaccharides in the activation of innate immunological mechanisms [reviewed in (Raetz and Whitfield, 2002; Kumar et al., 2009)]. In addition, the conjugation of polysaccharides to protein structures can generate hybrid molecules of increased immunogenicity. In fact, vaccines containing polysaccharides against several prokaryotic pathogens have proven successful and are commercially available (Pichichero, 2005; Pletz et al., 2008).

Polysaccharides are essential for pathogenic mechanisms and for the immune response during fungal infections (Roeder et al., 2004). Unlike mammalian cells, fungi have a cell wall, a complex compartment mainly composed of polysaccharides (Nimrichter et al., 2005). Glucans, chitin, and mannans (polymers consisting of repeating units of, respectively, glucose, $\mathrm{N}$-acetylglucosamine, and mannose) are particularly abundant in the fungal cell wall. Depending on their structural particularities, cell wall 
polysaccharides function as regulators of virulence or activators of innate immunity (Roeder et al., 2004; Zaragoza et al., 2009). Well characterized immunoactive polysaccharides produced by fungi include $\alpha$ - and $\beta$-glucans (Brown et al., 2003; Hohl et al., 2005; Bittencourt et al., 2006; Rappleye et al., 2007; Wheeler et al., 2008; van de Veerdonk et al., 2009; Chai et al., 2011) and complex mannans (Leitao et al., 2003; Cambi et al., 2008; van de Veerdonk et al., 2009). Candida albicans, Aspergillus fumigatus, Histoplasma capsulatum, Cryptococcus neoformans, and C. gattii are examples of fungal pathogens in which the immune functions of polysaccharides are known in great detail (Monari et al., 2006; Rappleye et al., 2007; Cambi et al., 2008; Chai et al., 2011).

Cryptococcus neoformans and C. gattii are the etiologic agents of cryptococcosis, a disease that presumably begins in the lung. In immunocompromised individuals, $C$. neoformans can disseminate to the central nervous system and other organs (Bicanic and Harrison, 2004). C. gattii, on the other hand, can cause disseminated disease in immunocompetent individuals (Byrnes et al., 2011). It is estimated that about one million new cases of cryptococcosis occur annually, with mortality rates that can reach 60\% (Park et al., 2009). In Brazil, cryptococcosis is the fungal disease with highest mortality rates among HIV-positive individuals (Prado et al., 2009). C. neoformans and C. gattii have polysaccharide capsules surrounding the cell body. Bacterial capsules are common in bacterial pathogens, but relatively rare and still poorly defined among eukaryotic organisms.

In C. neoformans and C. gattii, the capsule is mainly composed of the polysaccharides glucuronoxylomannan (GXM) and galactoxylomannan (GalXM; Zaragoza et al., 2009; Rodrigues et al., 2011). An alteration in the GalXM nomenclature to glucuronoxylomannogalactan (GXMGal) has been recently proposed based on structural aspects and monosaccharide composition (Heiss et al., 2009). The principal capsular polysaccharide component in both species is GXM, which consists of a polymer composed of an $\alpha 1,3$ linked mannosyl backbone with $\beta 1,2$ and $\beta 1,4$ xylosyl and glucuronyl substitutions (for details, see Zaragoza et al., 2009). Structural variation resulting from differences in composition, substitution, and conformation results in different serological properties. These GXM properties divide C. neoformans strains into four main serotypes: $A$ and D, produced by C. neoformans, and B and C, produced by C. gattii [review in (Zaragoza et al., 2009; Rodrigues et al., 2011)]. Hybrid serotypes have been also characterized in different regions (Xu et al., 2002). GXM is believed to be synthesized intracellularly, possibly in close association to lipid structures (Rodrigues et al., 2007; Oliveira et al., 2009). For construction of the capsule, yeast cells secrete polysaccharides into the extracellular environment by mechanisms that involve the release of vesicles for subsequent polysaccharide incorporation into the cell surface, for distal capsular enlargement (Zaragoza et al., 2006; Rodrigues et al., 2007).

It is assumed that synthesis of GXM and its release to the extracellular space are crucial for the immunopathogenesis of cryptococcosis. In general, GXM is deleterious to the immune system (Monari et al., 2006), although several reports indicate that this polysaccharide is a potent activator of the complement system and of the innate immunity [review in (Zaragoza et al., 2009)]. Fractions of extracellular GXM released into the medium were recently shown to differ in structure and function from capsular polysaccharide extracts (Frases et al., 2008). These observations suggested that C. neoformans and C. gattii cells produce highly diverse populations of GXM that show structural peculiarities with possible implications on their biological roles. In fact, various studies have established that the production of polysaccharides by $C$. neoformans includes GXM molecules with highly variable dimensions, which interact through several mechanisms to form the capsular network (Nimrichter et al., 2007; Frases et al., 2009). These studies raised an important question: do polysaccharide samples of identical composition but variable size and degree of polymerization present distinct biological functions?

The hypothesis raised above is supported by observations that have recently become available in the literature. Chitin, a linear polysaccharide found in fungi, crustaceous, insects, and parasites, is a water insoluble polymer composed of units of $\beta 1,4$-linked $N$-acetylglucosamine. As described by Da Silva et al. (2008, 2009), fractions of chitin with high molecular dimensions are immunologically inert. Polysaccharide samples with reduced dimensions, however, were associated with the effective stimulation of innate immunity and production of pro- and antiinflammatory cytokines. These observations have established a clear precedent in the literature indicating that polysaccharide samples of identical composition but with varying dimensions may have different functions.

The observation described above and the fact that capsular structures of Cryptococcus species are composed of GXM molecules of various sizes (Mcfadden et al., 2006; Frases et al., 2009) support the hypothesis that variation in these structures could translate into different biological effects. In fact, Fonseca et al. (2010) recently established a parallel between the functions of GXM fractions and their dimensions. Through the use of an experimental model that included tests of the activation of cellular responses resulting in nitric oxide production by phagocytes and activation of Toll-like receptors in epithelial cells, it was observed that GXM samples isolated from C. gattii (serotype B) with monosaccharide compositions that were similar to other polysaccharide fractions produced by C. neoformans (serotypes A and D) and even by other strains of C. gattii (serotype C) generated very distinct cellular responses. Measurements of polysaccharide diameter by dynamic light scattering (for details about the technique, see Frases et al., this issue) revealed that the increased capacity of C. gattii GXM to induce cellular responses was correlated with a reduced molecular diameter (Fonseca et al., 2010). These results led to the conclusion that serotype B GXM samples with reduced dimensions have greater immunobiological potential, as demonstrated for chitin (Da Silva et al., 2008, 2009; Lee et al., 2008). It remains unknown, however, whether this concept would be pertinent to other GXM-producing species, including members of the Trichosporon genus (Fonseca et al., 2009).

The findings discussed above imply new concepts about the structure and function of fungal polysaccharides. Besides structural aspects traditionally studied, such as sequence analysis, compositional determination, anomeric configuration, type of glycosidic linkage type, and presence of substituents, it becomes 
clear that other structural parameters, including molecular diameter and degree of polymerization, must be considered for functional studies. In fact, the conclusion that polysaccharide functions are influenced by these additional parameters was further supported by Cordero et al. (2011). Using static and dynamic light scattering, viscosity analysis, and high-resolution microscopy of $C$. neoformans polysaccharides, this study demonstrated that spatial conformation (branching) influences phagocytosis, nitric oxide production by macrophage-like cells, and susceptibility to reactive oxygen species, serology, and clearance during infection. These previously unexplored parameters can generate new insights, for example, on immunogenicity assays of polysaccharides, as well as their use in therapy or prevention of diseases. Studies in this area are still embryonic and, clearly, much remains to be discovered. For

\section{REFERENCES}

Bertozzi, C. R., and Rabuka, D. (2009). Structural basis of glycan diversity, in Essentials of Glycobiology, Chap. 2, 2nd Edn. Eds A. Varki, R. D. Cummings, J. D. Esko, H. H. Freeze, P. Stanley, C. R. Bertozzi, G. W. Hart, and M. E. Etzler, Cold Spring Harbor, NY: Cold Spring Harbor Laboratory Press.

Bicanic, T., and Harrison, T. S. (2004). Cryptococcal meningitis. Br. Med. Bull. 72, 99-118.

Bittencourt, V. C., Figueiredo, R. T., Da Silva, R. B., Mourao-Sa, D. S., Fernandez, P. L., Sassaki, G. L., Mulloy, B., Bozza, M. T., and BarretoBergter, E. (2006). An alpha-glucan of Pseudallescheria boydii is involved in fungal phagocytosis and toll-like receptor activation. J. Biol. Chem. 281, 22614-22623.

Brown, G. D., Herre, J., Williams, D. L., Willment, J. A., Marshall, A. S., and Gordon, S. (2003). Dectin1 mediates the biological effects of beta-glucans. J. Exp. Med. 197, 1119-1124.

Byrnes, E. J. III, Bartlett, K. H., Perfect, J. R., and Heitman, J. (2011). Cryptococcus gattii: an emerging fungal pathogen infecting humans and animals. Microbes Infect. doi: 10.1016/j.micinf.2011.05.009. [Epub ahead of print].

Cambi, A., Netea, M. G., Mora-Montes, H. M., Gow, N. A., Hato, S. V., Lowman, D. W., Kullberg, B. J., Torensma, R., Williams, D. L., and Figdor, C. G. (2008). Dendritic cell interaction with Candida albicans critically depends on $\mathrm{N}$ linked mannan. J. Biol. Chem. 283, 20590-20599.

Chai, L. Y., Vonk, A. G., Kullberg, B. J., Verweij, P. E., Verschueren, I., Van Der Meer, J. W., Joosten, L. A., Latge, J. P., and Netea, M. G. (2011). Aspergillus fumigatus cell wall components differentially modulate host TLR2 and
TLR4 responses. Microbes Infect. 13, 151-159.

Cordero, R. J., Frases, S., Guimaraes, A. J., Rivera, J., and Casadevall, A. (2011). Evidence for branching in cryptococcal capsular polysaccharides and consequences on its biological activity. Mol. Microbiol. 79, 1101-1117.

Da Silva, C. A., Chalouni, C., Williams, A., Hartl, D., Lee, C. G., and Elias, J. A. (2009). Chitin is a size-dependent regulator of macrophage TNF and IL-10 production. J. Immunol. 182, 3573-3582.

Da Silva, C. A., Hartl, D., Liu, W., Lee, C. G., and Elias, J. A. (2008). TLR-2 and IL-17A in chitin-induced macrophage activation and acute inflammation. J. Immunol. 181, 4279-4286.

Fonseca, F. L., Frases, S., Casadevall, A., Fischman-Gompertz, O., Nimrichter, L., and Rodrigues, M. L. (2009). Structural and functional properties of the Trichosporon asahii glucuronoxylomannan. Fungal Genet. Biol. 46, 496-505.

Fonseca, F. L., Nohara, L. L., Cordero, R. J., Frases, S., Casadevall, A., Almeida, I. C., Nimrichter, L., and Rodrigues, M. L. (2010). Immunomodulatory effects of serotype B glucuronoxylomannan from Cryptococcus gattii correlate with polysaccharide diameter. Infect. Immun. 78, 3861-3870.

Frases, S., Nimrichter, L., Viana, N. B., Nakouzi, A., and Casadevall, A. (2008). Cryptococcus neoformans capsular polysaccharide and exopolysaccharide fractions manifest physical, chemical, and antigenic differences. Eukaryot. Cell 7, 319-327.

Frases, S., Pontes, B., Nimrichter, L., Viana, N. B., Rodrigues, M. L., and Casadevall, A. (2009). Capsule of Cryptococcus neoformans grows by enlargement of polysaccharide

conceptual validation, future studies must include evaluation of the relationship between immunoactivity and molecular dimensions of polysaccharides synthesized by other organisms, as well as of fungal glycans other than GXM and chitin.

\section{ACKNOWLEDGMENTS}

Marcio L. Rodrigues and Leonardo Nimrichter are supported by grants from the Coordenação de Aperfeiçoamento de Pessoal de Nível Superior (CAPES, Brazil), Conselho Nacional de Desenvolvimento Científico e Tecnológico (CNPq, Brazil), Fundação de Amparo a Pesquisa do Estado de São Paulo (FAPESP,Brazil), and Fundação de Amparo a Pesquisa do Estado do Rio de Janeiro (FAPERJ, Brazil). Arturo Casadevall is supported by NIH grants AI033142, AI033774, AI052733, and HL059842.

molecules. Proc. Natl. Acad. Sci. U.S.A. 106, 1228-1233.

Heiss, C., Klutts, J. S., Wang, Z., Doering, T. L., and Azadi, P. (2009). The structure of Cryptococcus neoformans galactoxylomannan contains beta-D-glucuronic acid. Carbohydr. Res. 344, 915-920.

Hohl, T. M., Van Epps, H. L., Rivera, A., Morgan, L. A., Chen, P. L., Feldmesser, M., and Pamer, E. G. (2005). Aspergillus fumigatus triggers inflammatory responses by stage-specific beta-glucan display. PLoS Pathog. 1, e30. doi: 10.1371/journal.ppat.0010030

Kumar, H., Kawai, T., and Akira, S. (2009). Toll-like receptors and innate immunity. Biochem. Biophys. Res. Commun. 388, 621-625.

Lee, C. G., Da Silva, C. A., Lee, J. Y., Hartl, D., and Elias, J. A. (2008). Chitin regulation of immune responses: an old molecule with new roles. Curr. Opin. Immunol. 20, 684-689.

Leitao, E. A., Bittencourt, V. C., Haido, R. M., Valente, A. P., Peter-Katalinic, J., Letzel, M., De Souza, L. M., and Barreto-Bergter, E. (2003). Beta-galactofuranosecontaining O-linked oligosaccharides present in the cell wall peptidogalactomannan of Aspergillus fumigatus contain immunodominant epitopes. Glycobiology 13, 681-692.

Levitz, S. M. (2004). Interactions of tolllike receptors with fungi. Microbes Infect. 6, 1351-1355.

Mazmanian, S. K., and Kasper, D. L. (2006). The love-hate relationship between bacterial polysaccharides and the host immune system. Nat. Rev. Immunol. 6, 849-858.

Mcfadden, D. C., De Jesus, M., and Casadevall, A. (2006). The physical properties of the capsular polysaccharides from Cryptococcus neoformans suggest features for capsule construction. J. Biol. Chem. 281, 1868-1875.
Monari, C., Bistoni, F., and Vecchiarelli, A. (2006). Glucuronoxylomannan exhibits potent immunosuppressive properties. FEMS Yeast Res. 6, 537-542.

Moxon, E. R., and Kroll, J. S. (1990). The role of bacterial polysaccharide capsules as virulence factors. Curr. Top. Microbiol. Immunol. 150, 65-85.

Mulloy, B., Hart, G. W., and Stanley, P. (2009). Structural analysis of glycans, in Essentials of Glycobiology, Chap. 47, 2nd Edn. Eds A. Varki, R. D. Cummings, J. D. Esko, H. H. Freeze, P. Stanley, C. R. Bertozzi, G. W. Hart, and M. E. Etzler, Cold Spring Harbor, NY: Cold Spring Harbor Laboratory Press.

Nimrichter, L., Frases, S., Cinelli, L. P., Viana, N. B., Nakouzi, A., Travassos, L. R., Casadevall, A., and Rodrigues, M. L. (2007). Self-aggregation of Cryptococcus neoformans capsular glucuronoxylomannan is dependent on divalent cations. Eukaryot. Cell 6, 1400-1410.

Nimrichter, L., Rodrigues, M. L., Rodrigues, E. G., and Travassos, L. R. (2005). The multitude of targets for the immune system and drug therapy in the fungal cell wall. Microbes Infect. 7, 789-798.

Oliveira, D. L., Nimrichter, L., Miranda, K., Frases, S., Faull, K. F., Casadevall, A., and Rodrigues, M. L. (2009). Cryptococcus neoformans cryoultramicrotomy and vesicle fractionation reveals an intimate association between membrane lipids and glucuronoxylomannan. Fungal Genet. Biol. 46, 956-963.

Park, B. J., Wannemuehler, K. A., Marston, B. J., Govender, N., Pappas, P. G., and Chiller, T. M. (2009). Estimation of the current global burden of Cryptococcal meningitis among persons living with HIV/AIDS. AIDS 23, 525-530.

Pichichero, M. E. (2005). Meningococcal conjugate vaccines. Expert Opin. Biol. Ther. 5, 1475-1489. 
Pletz, M. W., Maus, U., Krug, N., Welte, T., and Lode, H. (2008). Pneumococcal vaccines: mechanism of action, impact on epidemiology and adaption of the species. Int. J. Antimicrob. Agents 32, 199-206.

Prado, M., Da Silva, M. B., Laurenti, R., Travassos, L. R., and Taborda, C. P. (2009). Mortality due to systemic mycoses as a primary cause of death or in association with AIDS in Brazil: a review from 1996 to 2006. Mem. Inst. Oswaldo Cruz 104, 513-521.

Raetz, C. R., and Whitfield, C. (2002). Lipopolysaccharide endotoxins. Annu. Rev. Biochem. 71, 635-700.

Rappleye, C. A., Eissenberg, L. G., and Goldman, W. E. (2007). Histoplasma capsulatum alpha$(1,3)$-glucan blocks innate immune recognition by the beta-glucan receptor. Proc. Natl. Acad. Sci. U.S.A. 104, 1366-1370.

Roberts, I. S. (1996). The biochemistry and genetics of capsular polysaccharide production in bacteria. Annu. Rev. Microbiol. 50, 285-315.

Rodrigues, M. L., Casadevall, A., and Zaragoza, O. (2011). "The architecture and antigenic composition of the polysaccharide capsule," in Cryptococcus: From Human Pathogen to Model Yeast, eds T. K. Joseph
Heitman, J. Kwon-Chung, J. Perfect and A. Casadevall (Washington, DC: American Society for Microbiology), 43-54.

Rodrigues, M. L., Nimrichter, L., Oliveira, D. L., Frases, S., Miranda, K., Zaragoza, O., Alvarez, M., Nakouzi, A., Feldmesser, M., and Casadevall, A. (2007). Vesicular polysaccharide export in Cryptococcus neoformans is a eukaryotic solution to the problem of fungal transcell wall transport. Eukaryot. Cell 6, 48-59.

Roeder, A., Kirschning, C. J., Rupec, R. A., Schaller, M., Weindl, G., and Korting, H. C. (2004). Toll-like receptors as key mediators in innate antifungal immunity. Med. Mycol. 42, 485-498.

Smith, H. (1990). Pathogenicity and the microbe in vivo. The 1989 Fred Griffith Review Lecture. J. Gen. Microbiol. 136, 377-393.

Stanley, P., and Cummings, R. D. (2009). Structures common to different glycans, in Essentials of Glycobiology, Chap. 13, 2nd Edn. Eds A. Varki, R. D. Cummings, J. D. Esko, H. H. Freeze, P. Stanley, C. R. Bertozzi, G. W. Hart, and M. E. Etzler, Cold Spring Harbor, NY: Cold Spring Harbor Laboratory Press.

van de Veerdonk, F. L., Marijnissen, R. J., Kullberg, B. J., Koenen, H. J., Cheng,
S. C., Joosten, I., Van Den Berg, W. B., Williams, D. L., Van Der Meer, J. W., Joosten, L. A., and Netea, M. G. (2009). The macrophage mannose receptor induces IL-17 in response to Candida albicans. Cell Host Microbe 5, 329-340.

Vecchiarelli, A. (2007). Fungal capsular polysaccharide and T-cell suppression: the hidden nature of poor immunogenicity. Crit. Rev. Immunol. 27, 547-557.

Weintraub, A. (2003). Immunology of bacterial polysaccharide antigens. Carbohydr. Res. 338, 2539-2547.

Wheeler, R. T., Kombe, D., Agarwala, S. D., and Fink, G. R. (2008). Dynamic, morphotype-specific Candida albicans beta-glucan exposure during infection and drug treatment. PLoS Pathog. 4, e1000227. doi: 10.1371/journal.ppat.1000227

Xu, J., Luo, G., Vilgalys, R. J., Brandt, M. E., and Mitchell, T. G. (2002). Multiple origins of hybrid strains of Cryptococcus neoformans with serotype AD. Microbiology 148, 203-212.

Zaragoza, O., Rodrigues, M. L., De Jesus, M., Frases, S., Dadachova, E., and Casadevall, A. (2009). The capsule of the fungal pathogen Cryptococcus neoformans. Adv. Appl. Microbiol. 68, 133-216.

Zaragoza, O., Telzak, A., Bryan, R. A., Dadachova, E., and Casadevall, A.
(2006). The polysaccharide capsule of the pathogenic fungus Cryptococcus neoformans enlarges by distal growth and is rearranged during budding. Mol. Microbiol. 59, 67-83.

Conflict of Interest Statement: The authors declare that the research was conducted in the absence of any commercial or financial relationships that could be construed as a potential conflict of interest.

Received: 15 July 2011; accepted: 28 July 2011; published online: 15 August 2011.

Citation: Rodrigues ML, Nimrichter $L$, Cordero RJB and Casadevall $A$ (2011) Fungal polysaccharides: biological activity beyond the usual structural properties. Front. Microbio. 2:171. doi: 10.3389/fmicb.2011.00171

This article was submitted to Frontiers in Fungi and Their Interactions, a specialty of Frontiers in Microbiology. Copyright (c) 2011 Rodrigues, Nimrichter, Cordero and Casadevall. This is an open-access article subject to a nonexclusive license between the authors and Frontiers Media SA, which permits use, distribution and reproduction in other forums, provided the original authors and source are credited and other Frontiers conditions are complied with. 